\title{
Plant Disease Detection using Internet of Thing (IoT)
}

\author{
Muhammad Amir Nawaz ${ }^{1}$, Tehmina khan ${ }^{2}$, Rana Mudassar Rasool ${ }^{3}$, Maryam Kausar ${ }^{4}$ \\ Amir Usman ${ }^{5}$, Tanvir Fatima Naik Bukht ${ }^{6}$, Rizwan Ahmad ${ }^{7}$, Jaleel Ahmad ${ }^{8}$ \\ Department of Computer Science, Institute of Southern Punjab Multan, Pakistan 1,2,3,4,6 \\ Department of Computer Science, NCBA\&E Multan, Pakistan ${ }^{5}$ \\ Department of Computer Science, Ripah International University Lahore, Pakistan ${ }^{7,8}$
}

\begin{abstract}
This paper presents the idea of internet of things (IOT) innovation to percept data, and talks about the job of the IOT innovation in farming infection and bug nuisance control, which incorporates rural ailment and bug checking framework, gathering sickness and creepy crawly bother data utilizing sensor hubs, information preparing and mining, etc. A malady and bug irritation control framework dependent on IOT is proposed, which comprised of three levels and three frameworks. The framework can give another approach to get to horticultural data for the farm. In this paper a computerized framework has been created to decide if the plant is ordinary or infected. The typical development of the plants, yield and nature of horticultural items is truly influenced by plant illness. This paper attempt to build up a robotized framework that identifies the nearness of disease in the plants. A mechanized ailment recognition framework is created utilizing sensors like temperature, moistness and shading dependent on variety in plant leaf wellbeing condition. The qualities dependent on temperature, mugginess and shading parameters are utilized to distinguish nearness of plant sickness.
\end{abstract}

Keywords-Plant diseases; internet of things; temperature sensor plants; farming

\section{INTRODUCTION}

\section{A. Background and Motivation}

Pakistan is a place that is known for agribusiness. Twothird of populace depends upon farming for their work. It is the fundamental establishment of financial improvement of the nation. The agribusiness likewise gives work chances to exceptionally huge level of populace.

Plant health condition assumes a crucial job to acquire great benefit for the ranchers. Legitimate checking of plant health is required at various phases of plant development so as to anticipate malady influencing plants. Presence of vermin and malady influence the estimation of harvest development and limits yield significantly. Present day framework relies upon unaided eye perception which is a tedious procedure. Programmed recognition of plant infection can be embraced to distinguish plant illness at beginning times. Different illness the board methodologies have been utilized by farmer at customary intervals so as to avoid plant diseases. Research in agriculture robots has been developing in the most recent years, on account of potential applications and industry endeavors in robot improvement. Their job was explored for some agrarian errands, chiefly engaged in expanding mechanization of traditional farming machines and making procedures, for example, progress planning, seeding, preparation, and reaping. Efficient, dreary, and time- subordinate errands appear to speak to the best fields of use for robots, particularly in an arable cultivating setting with brief harvests. Close to agronomic practices, automated plant assurance has likewise been explored, yet may speak to the most intricate test for analysts and engineers since inquiries in respect to pathogen determination must be considered alongside normal robot-related issues. As of late, look into in programmed acknowledgment of sicknesses has been quickly developing, with potential applications for creating robots ready to perceive single plants, find and distinguish ailments, and begin schedules for ailment the board. This paper expects to give subtleties of that new age of robots that could bolster plant pathologists.

\section{B. Internet of Things (IoT) in Plants}

The overall blueprint of Internet of things helper parts showed of mind blowing ability in those headways in spaces of plants and the continuous example of Accuracy in plants. Continuous improvements in sensor development, nearby downsizing in equipment in addition remarkable descent in their rate have donated the ton to a mechanical improvement of customary cultivating to correctness besides little scale exactness plants. Air sensor, ground sensor, radiation sensors, atmosphere station highlight that it is almost sensor as well as sensors data streams, which are secured as well as use for checking, data excavating, thinking, and control. Besides, starting late, there is a growing enthusiasm for high gauge as well as harmless country things. That example has produced the necessity for cover operable, spread, incredible, and careful co-appointments tractability systems. The IoT group of advances gives all the suitable instruments to building and keeping up such foundation and administrations, extraordinarily intended to help supply chains in rural and plant sectors Sensors, as wired and remote sensors, have been broadly utilized in horticulture during the most recent decades.

Detecting the earth in which creation happens, and, all the more as of late, the reactions of the plants to the atmosphere is urgent for taking the right and increasingly exact choices, streamlining profitability and nature of the cultivars. Adaptable gadgets, with high computational capacities, exceptionally advantageous structure factor and ease, would nowadays be able to be utilized, on batteries, and work for significant lots, with or without the help of intensity gathering modules. What's more, present day implanted gadgets have adequate assets to bolster all the more requesting sensors, for example, picture sensors, and the help of progressively modern systems administration conventions, such TCP/IP, broadening the customary systems administration abilities. 
Web of Stuffs is quickly progressing then various innovative presentations as well as organizations remain ascending out of that. The ton of exploration is actuality coordinating in the direction of joining a various dissimilar arrangements, Security insistence by numerous degrees of Internet of things as well as examination that will provide an unrivaled comprehension into the "Colossal Data" in order to improve distinctive business structures. National course of action of governments around the world for extended creation pace of fresh cut vegetables and meat, at lower cost, with higher quality benchmarks, similarly as, the buyers' enthusiasm for straightforwardness in the age cycle and the biological impression of the things they buy, give IoT a tremendous field to progress and scattering. As shown by Bradley the examinations from 2015-2020 of potential IoT worth vary basically, running from at any rate $\$ 1$ trillion up to more than $\$ 15$ trillion, barring the extended salaries, the benefits of cost decline among associations and adventures and the general budgetary development due to IoT. A huge piece of the extra estimation of IoT starts from the versatility and the improvement and precision that it brings into the age methodology of industry and creation units of various sorts. Subsequently, it isn't so perilous to check that cultivating zone shapes at all levels will profoundly change as soon as possible. Concerning agribusiness, IoT is required to streamline the age by various strategies. Farmlands and nurseries are going to move from precision to a littler scale exactness model of country creation. Disseminated, inescapable registering and exact observing of the offices will give the ideal developing or living conditions for the two vegetables and creatures. Selfruling frameworks will be capable not exclusively to direction the actuators in the most proficient manner, improving the utility and asset utilization, yet additionally to control the generation in understanding for a market circumstance, boosting the benefit as well as limiting expenses each technique under sun. Then again, sustenance supply chains, furnished with RFID gear, will most likely screen each phase in the life of an item, make programmed thinking, in the event of a broken item and increment customer's sentiment of wellbeing, through a straightforward item life cycle data framework.

Clearly the financial numbers identified with IoT are huge, enticing some intense players to put resources into it. Models, corresponding the progressing acquisition for Nest Laboratories, an association increasing pragmatic involvement of IoT in home computerization, through Google is dollar 4.02 billion in genuine money and the acquisition for Jasper Machineries, architect of as well as IoT will arrange, through Cisco with dollar 2.04 billion, reveal staggering ability for IoT as well as exhibit which is exceedingly speaking to tremendous money related experts and behemoth mechanical firms. The association course of action, regardless, isn't so irrelevant. This is because of the way that the organizations associated with IoT put resources into one or a couple of parts of it, due to its wide nature. Accordingly, at some point or another, they should collaborate with one another, setting aside any challenge, or the idea of who is increasingly significant, so as to present some widespread models in the advancing IoT publicity.

\section{IoT in Plant Disease Detection}

Identification of diseases in the plant is most extreme requirement for farmers and agriculture specialists. The principle point of the proposed framework is to recognize plant diseases with the help of IoT (Internet of Things). In the greater part of the plants the sickness beginning happens on plant leaves. Subsequently, in the proposed work we have considered location of plant sickness present on leaves. The segregation of ordinary and influenced plant leaf can be estimated dependent on variety in temperature, dampness and shading.

The pigments in leaves are in charge of the striking shading changes in the fall. Temperature, daylight and soil dampness all assume a job in how the leaves will look in the fall. Rich daylight and low temperatures after the abscission layer structures cause the chlorophyll to be demolished all the more quickly. We have utilized DHT11 temperature sensor. The DHT11 sensor detects the temperature of the leaf under thought. The parameters that are gathered from the sensor are sent to the cloud stage through the wifi shield associated with the Arduino UNO board. The information which is recorded for investigation in the cloud stage. We at first record the scope of the temperature of a sound leaf. Afterward, if the temperature of the leaf under thought does not fall into that run, at that point the leaf is said to be unhealthy.

Changes in the shade of plant tissue are a typical indication of plant disease. Regularly these shading changes are realized by the yellowing of typical green tissue because of the annihilation of chlorophyll or an inability to frame chlorophyll. Such suppression of leaf shading might be finished or halfway. The shading sensor detects the shade of the leaf under thought which is another parameter that is being utilized to decide if the leaf is either disease or safe.

As indicated by the normal for rural data stream, from the side of innovation, on account of the characteristics of by and large sense, solid exchange and wise procedure, IOT starts to turn into the primary strategy for information securing and transmission and would turn into a significant innovation over a few sorts of sensors to gather, investigate, transmit and deal with the entire information identified with plant illness and creepy crawly pests. The sensor is a significant innovation for information obtaining, and is principally used to catch some portion of the current information, relate and synchronize these information, examine them, lastly, does a receptive movement without client mediation. The parts of a (remote) detecting hub incorporate the accompanying: detecting and in citation unit (single component or exhibit), preparing unit, correspondence unit, control unit and other applicationsubordinate units Sensors can be basic point components be multi point location clusters, and it has the capacity of huge scale arrangement, low support, scale capacity, flexibility for various situations.

\section{LiteratuRE REVIEW}

This paper shows minimal effort shading sensors for checking plant development in a research facility. A mechanized framework for estimating plant leaf shading is created to check plant wellbeing status [1] by introducing 
minimal effort shading sensors for checking plant development in a research center. A computerized framework for estimating plant leaf shading is created to check plant wellbeing status [2]. This paper have exhibited novel calculation for division and programmed distinguishing proof of vermin on plants utilizing picture handling. The proposed system includes decreased computational intricacy and goes for irritation recognition in a nursery domain as well as in a ranch situation too. The whitefly, a bio-assailant which represents a danger to a huge number of yields, was picked as the nuisance of enthusiasm for this paper. The calculation was tried for a few whiteflies influencing various leaves and a precision of $96 \%$ of whitefly identification was accomplished. We have exhibited irritation control in rural ranches utilizing picture preparing strategies in MATLAB. Pictures are then exposed to pre-handling, change and bunching [3]. This paper has exhibited IoT Implementation for remote checking of agrarian parameters. Remote framework is created to screen natural conditions in horticulture field like temperature, soil $\mathrm{pH}$, soil wet level and stickiness adjacent to leaf sicknesses discovery [4]. We have introduced small scale controller based auto-water system and nuisance recognition utilizing picture handling. A technique for picture examination can widely connected to farming learning for stretch most extreme security of plants which can at last lead to all the more likely yield the executives and generation [5]. Plant diseases and creepy crawly vermin have transformed difficulty by way of which foundation substantial decrease of equally superiority and amount for crop items [6]. In this research we have introduced plan and advancement of vermin observing framework for actualizing exactness agribusiness utilizing IoT. Pakistan the greater part of the rancher develop sugarcane yet did not get yielding because of bugs and hatchlings in sugarcane. In this proposed structure framework utilized arduino for checking the clamor and temperature [7]. This paper has exhibited arduino based vermin control utilizing constant ecological observing sensors. This paper endeavors to build up a robot fit for performing task of apportioning nuisance control specialists, deterrent shirking for selfdirection on the field with no client impedance and make a sterile situation for the ideal development of the harvests in a continuous checked shut condition [8]. This paper has displayed a genuine nature sensor and reasonable assessment calculation for plant acknowledgment. The framework created depends on free and programmable real nature sensors for constant acknowledgment and distinguishing proof of individual weed and harvest plants utilizing numerical calculations and choice models [9]. This research has introduced apple leaf sickness distinguishing proof utilizing hereditary calculation and connection based element determination strategy. A shading change construction of information RGB picture remained planned right off the bat and after that RGB structure has been changed over to HSI. YUV as well as dim structure. Foundation has evacuated and afterward the illness spot picture was sectioned with district developing calculation (RGA). At last, the sicknesses were perceived by SVM classifier [10]. The brisk progression of new advances and the changing scene of the online world. Web of Things (IoT), Internet of All, cloud-based plans) give a novel opportunity to making modernized and mechanical systems for urban developing, cultivating, and officer administration. Creative advances in machine vision, overall arranging structures, laser advances, actuators, and mechatronics have engaged the improvement and use of mechanical systems and smart advances for precision agribusiness. In this, we present and review mechanical applications on plant pathology and the board, and rising agricultural developments for intra urban cultivating. Nursery impelled the official's structures and headways have been essentially made in the latest years, joining IoT and WSN (Wireless Sensor Network). AI, machine vision, and AI (Artificial Intelligence) have been utilized and associated in agriculture for robotized and mechanical developing. Understanding advancements, using machine vision/learning, have been made not only for planting, water framework, weeding (to some degree), pruning, and procuring, yet moreover for plant ailment acknowledgment and recognizing confirmation. Regardless, plant disorder recognizable proof still addresses a fascinating test, for both abiotic and biotic weight. Various affirmation procedures and advances for perceiving plant disease reactions have been successfully developed; still, the vast majority of them require a controlled area for data getting to dodge false positives. Man-made intelligence techniques (e.g., significant and move learning) present promising results for improving picture dealing with and plant reaction recognizing evidence. Before long, demonstrative expressness is a test for microorganism control and should drive the improvement of mechatronics and mechanical responses for ailment the board [11].

\section{MATERIALS AND MethodOlOGY}

The proposed framework comprises of temperature, dampness, and shading sensors for gathering information from plant leaves dependent on variety in temperature, mugginess and shade of plant leaves. The information gathered from the leaves comprises of current ecological variables like temperature, moistness and shading. The progressions that a plant experiences are caught by the

Temperature moistness and shading sensors and dissected with the Arduino programming. The information gathered with temperature, moistness as well as shading sensor are specified for Arduino UNO unit after that data remains conveyed the ranchers. The framework utilizes WiFi shield so as to send the information from the host framework to the cloud stage for examination. The gathered information in the cloud stage is then contrasted with the dataset all together with identify whether the leaf under thought is typical or influenced. The Figure.1 indicates schematic chart of the proposed work. Step by Step explanation of figure 2 .

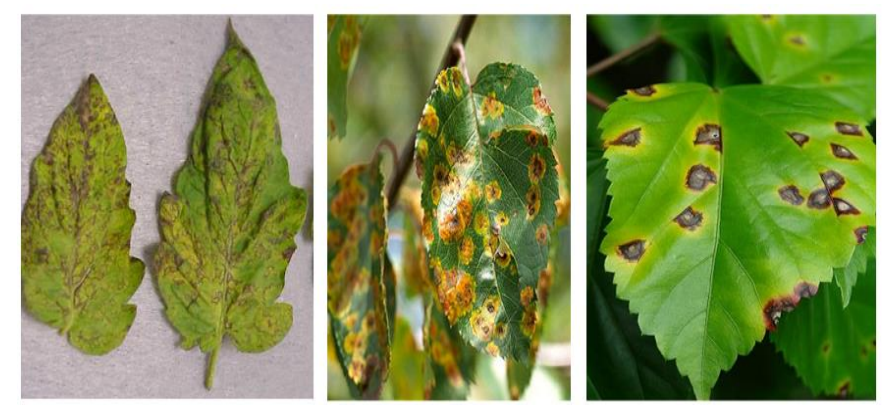


Fig. 1. Plant Leave Affected by Disease.

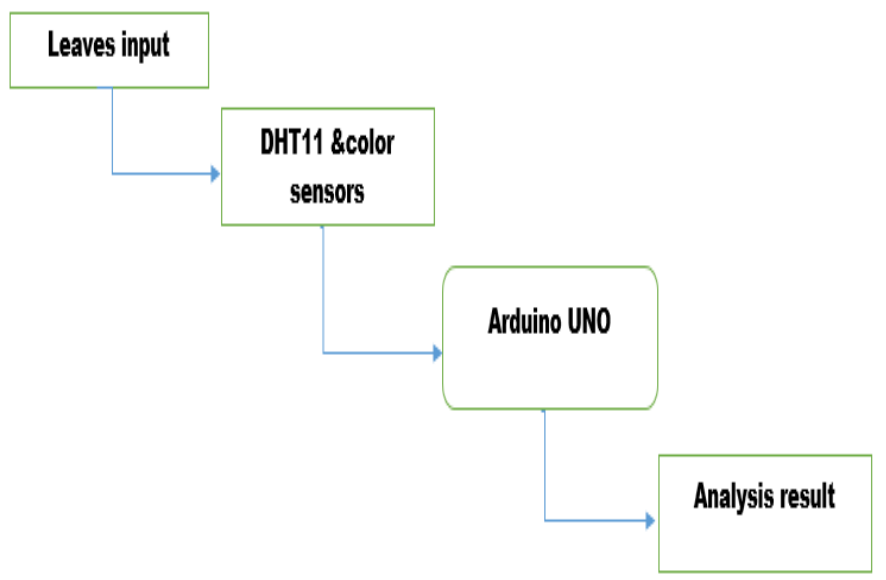

Fig. 2. Proposed Methodology.

1) Information obtaining: Here we accept tests of various leaves as the information. These leaves are then detected by the sensors to decide various parameters dependent on which it is perceived to be sound or infected.

2) Temperature sensors: The DHT11 is a fundamental, ultra simplicity modernized temperature sensor. It uses a capacitive moisture sensor and discharges a propelled sign on the data stick (no straightforward information pins required.

3) Humidity sensor: according figure 3 The DHT11 is a key, ultra simplicity propelled suddenness' sensor. It uses a capacitive moistness sensor and a thermistor to measure the incorporating air, and discharges an automated sign on the data stick.

4) Color Sensor: according figure 4 The TCS3200 is a programmable shading light-to-recurrence converter/sensor. The sensor is a solitary solid CMOS incorporated circuit that consolidates a configurable silicon photodiode and a currentto-recurrence converter.

5) Aurdino: according figure 5 The Arduino United Nation Organization is an comprehensively use open source controller board dependent on the ATmega330P microcontroller as well as prepared by Arduino.cc.

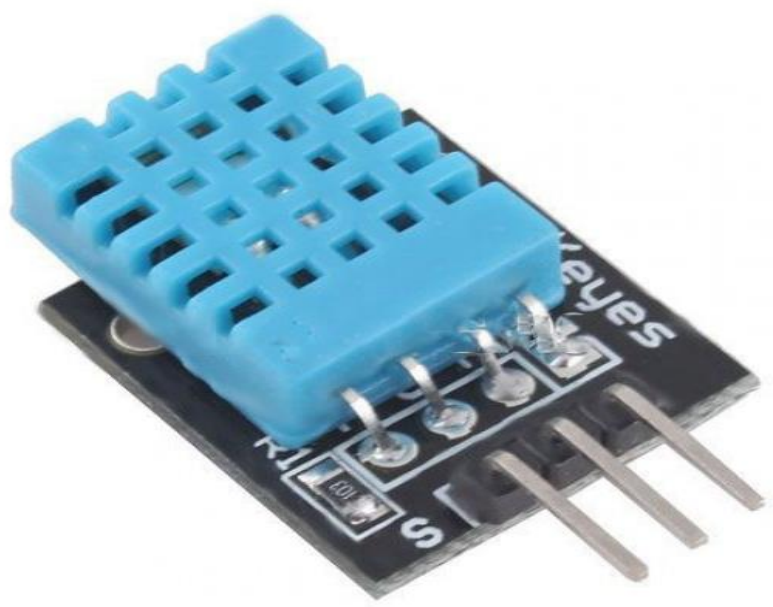

Fig. 3. DHT11 for Humidity and Temperature.

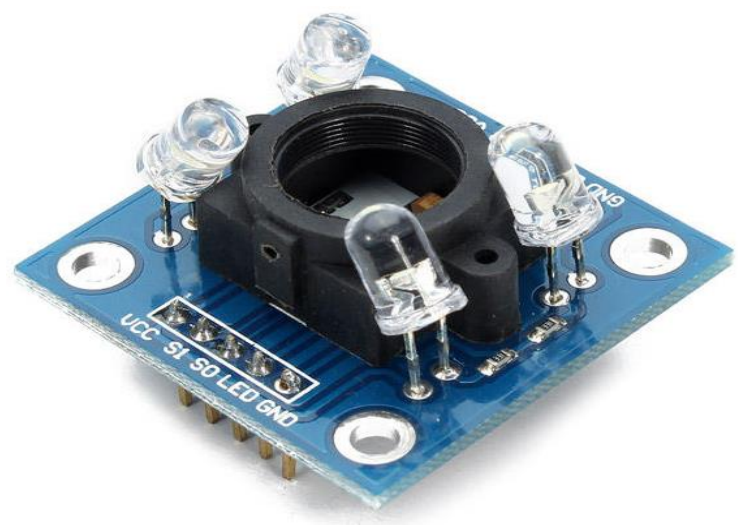

Fig. 4. Tcs3200 for get Color from Leaf.

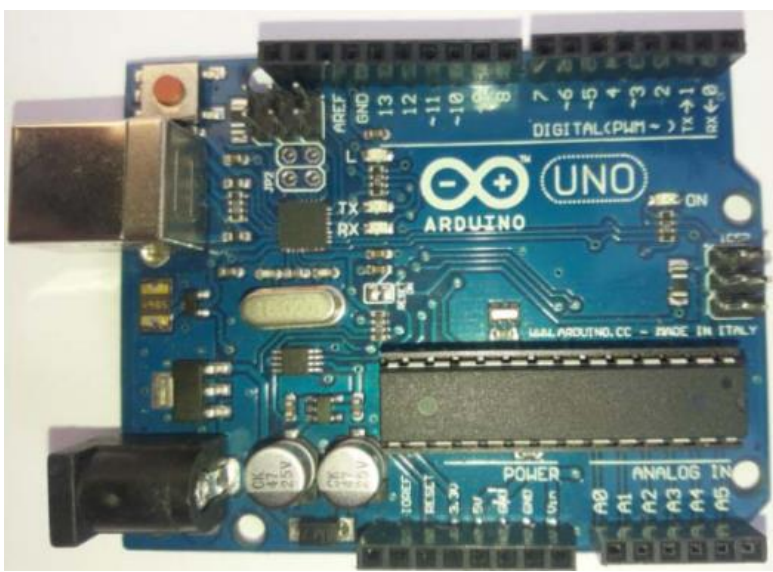

Fig. 5. Arduino used for Decision.

6) Cloud stage: Here we utilize "ThingSpeak" cloud stage to send the detected information to the cloud. This information sent is plotted against the diagram to see the adjustment in the temperature, mugginess and the shading. Contingent upon the information that is plotted against the diagram we check whether the qualities fall into a similar range. On the off chance that they do as such, at that point the leaf is sound or else it is sick

Algorithm 1: Identification of plant disease using temperature and color sensor

Input: leaf (infected or Normal)

Output: Normal or diseased plant leaf

Description: Given temperature range for the leaf to be healthy is $15-300 \mathrm{C}$

Start

Step 1: Get leaf for acquisition.

Step 2: measure temperature and color of the leaf using the DHT11 and TCS3200

sensor.

Step 3: calculate the color and temperature

if (minimum range $<$ temperature $<$ maximum range AND minimum range $<$ color $<$ maximum range)

Display "Leaf is Normal"

else 
Display "Leaf is Diseased"

Stop

\section{CONCLUSION}

In this paper, a framework is created to decide to the nature of the leaves. The proposed strategy utilizes the sensor gadgets to recognize the parameters like temperature, stickiness and shade of the leaves, which are then contrasted with the informational index with check whether the gathered qualities falls in to the range determined in the informational collection. The proposed model can be utilized in various territories by ranchers, industrialists, botanists, nourishment designers and doctors. The roads for further work here is the point to utilize the picture handling methods alongside the proposed framework to make it progressively proficient and furthermore exact to decide the qualities and to characterize whether the leaves are unhealthy or sound. To assemble an allencompassing form of the framework, we can utilize the picture preparing system that identify the sort of the sickness the leaf is influenced with and groups the various infections among the leaves. Here we can fabricate a mechanized framework with the goal that it is valuable for the enormous scale creation and furthermore helps in early discovery of the sicknesses that helps the customers for the better execution and upgrades the harvest yield.

The proposed framework is constrained to just identify whether the leaf under thought is solid or infected. This can be additionally done for perceiving the sort of infections in the leaves and arrangement of those ailments. We have constrained our work to just to the temperature, mugginess and shading parameters of the leaves. This can be additionally upgraded by applying different sensors and consolidating with picture preparing ideas. The other confinement is that the decided qualities for the considered parameters are not exact. We have taken the scope of qualities for those parameters and the range may fluctuate dependent on the climatic conditions.

\section{REFERENCES}

[1] Seelye, M., et al. Low cost colour sensors for monitoring plant growth in a laboratory. in 2011 IEEE International Instrumentation and Measurement Technology Conference. 2011. IEEE.

[2] Huddar, S.R., et al. Novel algorithm for segmentation and automatic identification of pests on plants using image processing. in 2012 Third International Conference on Computing, Communication and Networking Technologies (ICCCNT'12). 2012. IEEE.

[3] Krishnan, M. and G. Jabert, Pest Control in Agricultural Plantations Using Image Processing. IOSR Journal of Electronics and Communication Engineering (IOSR-JECE), 2013. 6(4): p. 68-74.

[4] SV, M.G. and S. Galande, Measurement of NPK, temperature, moisture, humidity using WSN. 2015.

[5] Rahman, S.A., et al., Reaction Decoder Tool (RDT): extracting features from chemical reactions. Bioinformatics, 2016. 32(13): p. 2065-2066.

[6] Hiary, H., et al., Fast and accurate detection and classification of plant diseases. International Journal of Computer Applications, 2011. 17(1): p. 31-38.

[7] Veerapandi, S., S. Gavaskar, and A. Sumithra, A Hybrid Fault Tolerance System for Distributed Environment using Check Point Mechanism and Replication. International Journal of Computer Applications, 2017. 975: p. 8887.

[8] Sai, V.V. and T. Hemalatha. Computational methods for simulating soil parameters using electrical resistivity technique. in 2017 8th International Conference on Computing, Communication and Networking Technologies (ICCCNT). 2017. IEEE.

[9] Schneider, F., et al., The effect of deep tillage on crop yield-What do we really know? Soil and tillage research, 2017. 174: p. 193-204.

[10] Chuanlei, Z., et al., Apple leaf disease identification using genetic algorithm and correlation based feature selection method. International Journal of Agricultural and Biological Engineering, 2017. 10(2): p. 7483.

[11] Ampatzidis, Y., L. De Bellis, and A. Luvisi, iPathology: robotic applications and management of plants and plant diseases. Sustainability, 2017. 9(6): p. 1010. 